\title{
Congenital panhipopituitarism and ectopic posterior pituitary
}

Iconaru Laura, Baleanu Felicia, Bourmorck Carole, Karmali Rafik CHU Brugmann, Bruxelles

Congenital hypopituitarism may be the result of complications linked with delivery or may sometimes result of insufficient development of the gland in the context of specific genetic abnormalities(PROK2 and PROKR2, LHX4, HESX1, OTX2, GLI2 and SOX3). Interruption or lack of pituitary stalk represents a frequent feature of congenital hypopituitarism.

\section{Case description}

We present a patient 39 years old with congenital panhypopituitarism, mental retardation, dysmorphic syndrome and diabetes type 1 but without diabetes insipidus.

MRI imaging showed ectopic posterior pituitary at the tuber cinereum level associated with the lack of pituitary stalk.

Medical management consists of replacement therapy for all lines deficient hormones and insulin therapy.

\section{Results}

Laboratory findings - baseline tests have demonstrated combined multiple pituitary hormone deficiency involving growth hormone, thyrotropin, adrenocorticotropic and gonadotropics hormones without response to dynamics tests (insulin tolerance test and releasing factors $\mathrm{TRH}, \mathrm{GnRH}$ and $\mathrm{CRH}$ ).

\section{Baseline tests}

\begin{tabular}{ll} 
GH & $<0.5 \mathrm{ng} / \mathrm{ml}$ \\
IGF1 & $35 \mathrm{ng} / \mathrm{ml}$ \\
TSH & $1.41 \mathrm{mU} / \mathrm{l}$ \\
fT4 & $0.4 \mathrm{ng} / \mathrm{dl}$ \\
fT3 & $1.2 \mathrm{pg} / \mathrm{ml}$ \\
ACTH & $12.2 \mathrm{pg} / \mathrm{ml}$ \\
Cortisol & $2.1 \mu \mathrm{g} / \mathrm{dl}$ \\
\hline LH & $<0.2 \mathrm{mU} / \mathrm{ml}$ \\
FSH & $<1 \mathrm{mU} / \mathrm{ml}$ \\
\hline Free Testosteron & $0.28 \mathrm{ng} / \mathrm{dl}$
\end{tabular}

\section{Dynamics tests}

\begin{tabular}{l|l|l|l|l|l|l|}
\hline Test & $0^{\prime}$ & $30^{\prime}$ & $60^{\prime}$ & $90^{\prime}$ & $120^{\prime}$ \\
$\begin{array}{l}\text { With } \\
\text { GHRH }\end{array}$ & $\begin{array}{l}\text { GH } \\
(\mathrm{ng} / \mathrm{ml})\end{array}$ & $<0.5$ & $<0.5$ & $<0.5$ & $<0.5$ & $<0.5 \mathrm{I}$ \\
\hline $\begin{array}{l}\text { With } \\
\text { TRH }\end{array}$ & $\begin{array}{l}\text { TSH } \\
(\mathrm{mU} / \mathrm{l})\end{array}$ & 0.03 & 0.15 & 0.14 & 0.11 & 0.10 \\
\hline $\begin{array}{l}\text { With } \\
\text { CRH }\end{array}$ & $\begin{array}{l}\text { ACTH } \\
(\mathrm{pg} / \mathrm{ml})\end{array}$ & 18.9 & 17.8 & 18.8 & 16.6 & 18.9 \\
\hline
\end{tabular}

$\begin{array}{llllll}\text { Cortisol } & 2.2 & 2.2 & 1.9 & 1.4 & 1\end{array}$ $(\mu \mathrm{g} / \mathrm{dl})$

With LH $\quad<0.2<0.2<0.2<0.2<0.2$

GnRH (mU/ml)

\begin{tabular}{llllll}
$\underset{(\mathrm{mU} / \mathrm{ml})}{\mathrm{FSH}}$ & 0.2 & 0.3 & 0.2 & 0.4 & 0.5 \\
\hline
\end{tabular}

MRI pituitary showed ectopic posterior pituitary at the tuber cinereum level associated with the lack of pituitary stalk.

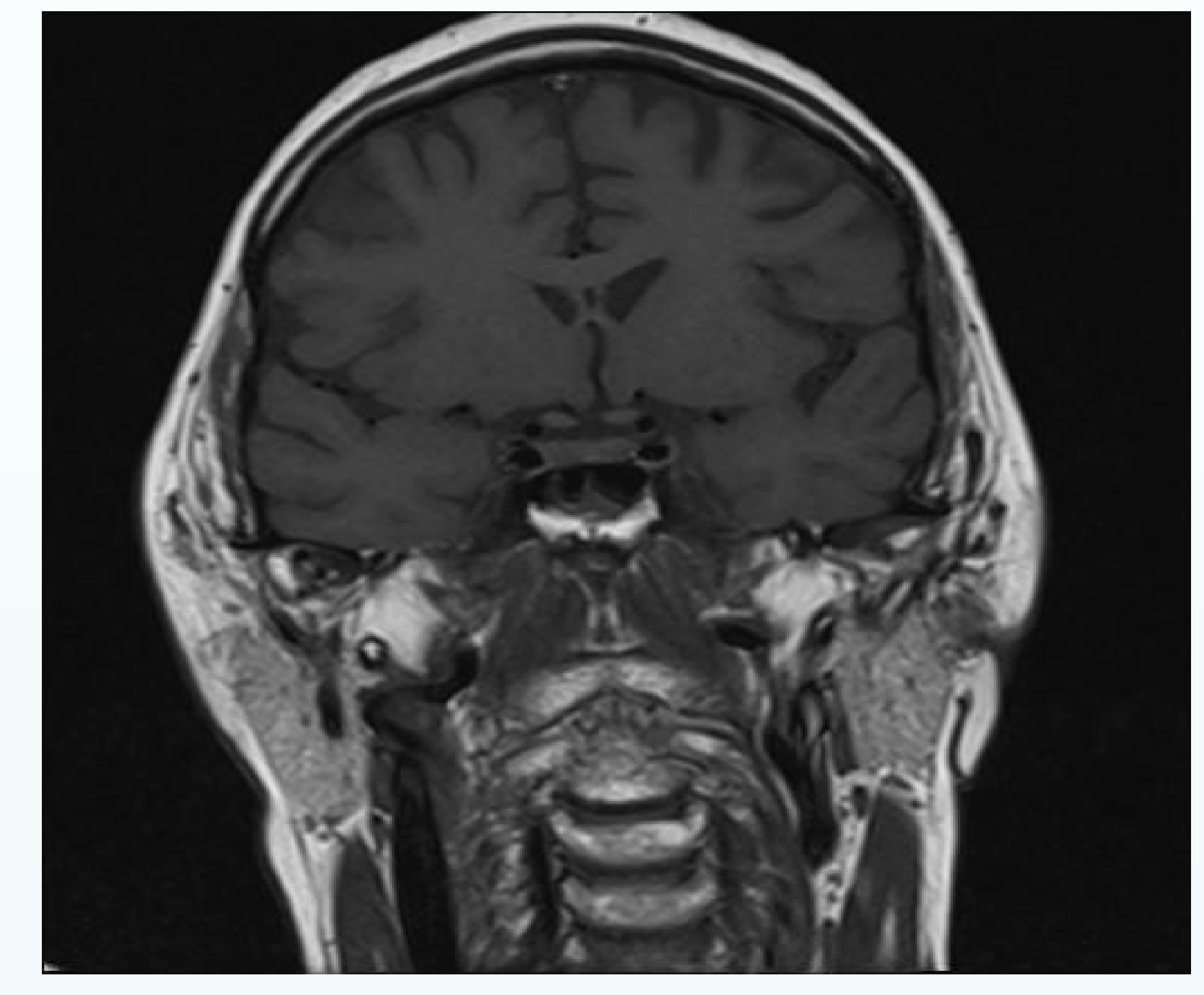

MRI pituitary

\section{DXA}

BMD lumbar spine $(L 2-L 4)=0,641$ $\mathrm{g} / \mathrm{cm} 2$,

Z- score $=-3,8 D S, T$-score $=-4,1 \mathrm{DS}$. BMD right hip $=0,432 \mathrm{~g} / \mathrm{cm} 2$,

Z- score $=-3,7 \mathrm{DS}, \mathrm{T}$-score $=-4,0 \mathrm{DS}$.

Treatment

Medical management consists of replacement therapy for all lines deficient hormones and insulin therapy.

\section{Conclusion}

The patients with no visible pituitary stalk on MRI present a more severe form of the disease associated with multiple deficiency of the anterior pituitary hormones, whereas the presence of the pituitary stalk leads to isolated GH deficiency. Follow-up on these patients is necessary, as the natural history of the disease is not established until adulthood.

\section{Key words}

Congenital hypopituitarism , pituitary stalk , ectopic posterior pituitary 